\title{
An Unsupervised and Drift-Adaptive Spike Detection Algorithm Based on Hybrid Blind Beamforming
}

\author{
Michal Natora and Klaus Obermayer \\ Institute for Software Engineering and Theoretical Computer Science, Faculty IV, Berlin Institute of Technology (TU Berlin), \\ Franklinstraße 28/29, 10623 Berlin, Germany \\ Correspondence should be addressed to Michal Natora, natora@cs.tu-berlin.de
}

Received 15 June 2010; Revised 25 October 2010; Accepted 16 November 2010

Academic Editor: Raviraj S. Adve

Copyright ( $) 2011$ M. Natora and K. Obermayer. This is an open access article distributed under the Creative Commons Attribution License, which permits unrestricted use, distribution, and reproduction in any medium, provided the original work is properly cited.

\begin{abstract}
In the case of extracellular recordings, spike detection algorithms are necessary in order to retrieve information about neuronal activity from the data. We present a new spike detection algorithm which is based on methods from the field of blind equalization and beamforming and which is particularly adapted to the specific signal structure neuronal data exhibit. In contrast to existing approaches, our method blindly estimates several waveforms directly from the data, selects automatically an appropriate detection threshold, and is also able to track neurons by filter adaptation. The few parameters of the algorithm are biologically motivated, thus, easy to set. We compare our method with current state-of-the-art spike detection algorithms and show that the proposed method achieves favorable results. Realistically simulated data as well as data acquired from simultaneous intra/extracellular recordings in rat slices are used as evaluation datasets.
\end{abstract}

\section{Introduction}

Extracellular recordings with electrodes constitute one of the main techniques for acquiring data from the central nervous system in order to study the neuronal code. Information in this system is transmitted by short electric impulses, called action potentials or, hereinafter, spikes. One of the first processing stages of the recorded data, hence, consist of identifying the occurrence times of these spikes. To this end, various spike detection algorithms have been developed. To give a structured overview of the recent development in this field, we use a categorization scheme based on the working principle of the methods. Note that although the spike detection stage is one of the earliest, basically all algorithms require already some preprocessing. This includes a band pass filtering (usually between $0.5 \mathrm{kHz}$ and $10 \mathrm{kHz}$ ) and a zero mean normalization. In the following, we will still refer to this kind of preprocessed data as "raw" data, since all techniques rely on this initial step.

The first category of spike detection methods assumes that the spikes exhibit a larger amplitude than noise fluctuations. Hence, spikes can be detected as data segments which amplitude cross a certain threshold value. In [1] three different variations of this detection paradigm were described, including maximum, minimum, and absolute value thresholding. Other related approaches rely on the distance between the minimum and maximum value within a certain time frame [2] or temporally hierarchical maximum and minimum value thresholding [3].

The principle of the second category is based on the transient nature of a spike; thus, spikes can be detected by measuring some quantity describing the discontinuity of data. An example is the nonlinear energy operator which takes into account instantaneous energy and frequency, and which was used for spike detection in [4]. Further adaptations of this method to neural data have been proposed in $[5,6]$. On the other hand, the approach in [7] considers only the instantaneous energy difference while the proposed method in [8] calculates the derivative of a temporally accumulated energy. Also based on the first derivate of the data are methods presented in $[9,10]$.

The algorithms falling into the third category rely on the fact that spikes from a specific neuron exhibit a characteristic waveform. The similarity between a data segment and 
a specified waveform decides whether the considered data segment contains a spike. When the actual waveform in the data is unknown, a generic approach can be used. For example in $[11,12]$ a biorthogonal, respectively, a coiflet mother wavelets are used, since they exhibit a certain similarity in shape to waveforms found in some real recordings, and a spike is said to be detected when a specific function of wavelet coefficients exceeds a threshold value. In contrast, unsupervised estimation (also called blind estimation) of the waveform or blind equalization has been performed in [13] by linear prediction, in [14] by automatic threshold setting, or in $[15,16]$ by using the cepstrum of bispectrum.

The choice which algorithm should be used in an application surely depends on the two important aspects of computational complexity and detection performance. Limited power and computing recourses, as encountered in implantable circuits [17], restrict applicable algorithm to have a very low computational load; hence most methods from the first category, and some few from the second one are used. When not limited by such constraints, it is favorable with respect to the detection performance to use algorithms belonging to the third category. This is motivated by the fact that given the waveform and the noise covariance matrix, the matched filter, or equivalently the minimum variance distortionless response beamformer (MVDR), is the optimal detector in case of Gaussian noise [18].

The aforementioned spike detection methods based on blind equalization suffer from three main drawbacks. Firstly, they construct only a single filter. In many experimental situations, however, spikes from more than one neuron, having distinct waveforms, are present in the electrode recordings. The single filter either captures just one waveform, meaning that spikes from the other neurons will be detected poorly, or the filter is an average filter which will have a suboptimal response to spikes from all the neurons. This problem aggravates that the more neurons are present, the more the waveforms are distinct, which is especially the case in multichannel recording devices, such as tetrodes [19].

Secondly, few methods offer an automatic threshold selection mechanism, thus allowing for a truly unsupervised operation. The available approaches [20-23] focus on the case when spike detection is done by amplitude thresholding (first category). For the above mentioned methods which rely on blind equalization, none or only heuristic values are given regarding the choice of an appropriate threshold.

Thirdly, the mentioned methods are nonadaptive. Once a filter is calculated on a data segment in the time interval $[t, t+T]$, it is also applied to all subsequent data segments at times $\tau>t+T$. Particularly in acute recordings, the shape of the waveform will change over time [24]; hence the performance of the filter will be suboptimal if it is not adapted. One could reestimate the template and recalculate the filter after every time interval; however, this would increase the computational load significantly, and tracking of neurons would become difficult.

In this contribution, we propose a new spike detection algorithm which overcomes all those drawbacks. The algorithm is derived by considering the spike detection task as a blind equalization problem in a multiple-input,

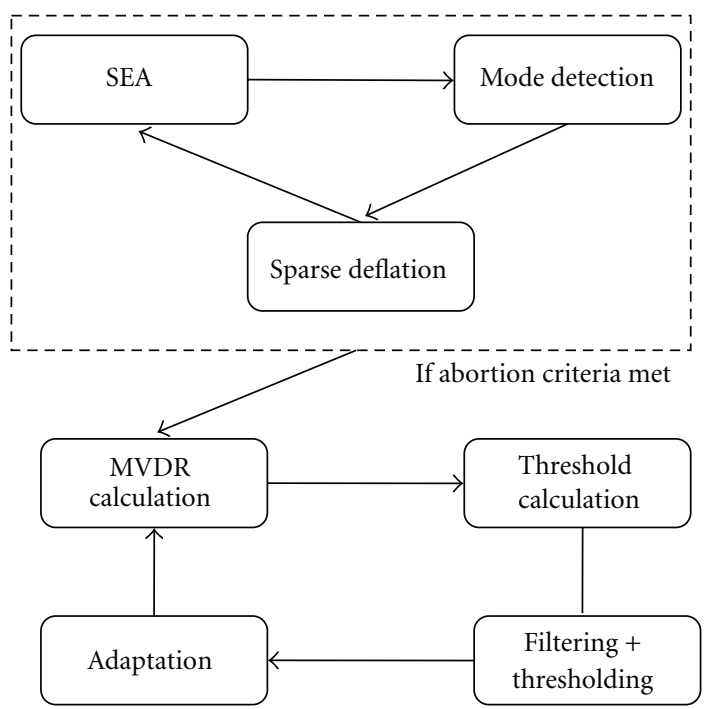

FIGURE 1: Schematic illustration of the proposed algorithm HBBSD. The algorithm starts with the superexponential algorithm (SEA) and iterates between SEA, Mode detection, and Sparse deflation repetitively, until certain abortion criteria described in Section 2.5 are met. This iterative procedure allows to estimate blindly several spike waveforms and the noise covariance matrix. Finally, the MVDR filters and the corresponding thresholds are calculated. Spike detection is done by thresholding the filter output and the newly detected spikes are used to update the filters, allowing for neuron tracking.

single-output system. The algorithm consists of a two-step procedure. In the first step, an iterative algorithm based on higher-order statistics, mode detection, and deflation is used. This gives estimates of the spike templates and the noise covariance matrix, from which in the next step, the minimum variance distortionless response (MVDR) beamformers are calculated, leading to an increased detection performance. This also allows to formulate a threshold selection algorithm as well as an effective adaptation scheme (see Figure 1 for a graphical representation of the whole algorithm). Because we use techniques from both fields, that is, blind equalization and classical beamforming, in the context of spike detection, we call our method hybrid blind beamforming for spike detection (HBBSD).

A simplified version of the core algorithm and some preliminary results were published in [25]. This contribution not only extends both in several ways but also presents a significant amount of new algorithms and results. Amongst others, the threshold calculation and the drift adaptation are introduced, and the algorithm is tested on many new datasets, including experimental data from rat tissue.

The rest of the paper is organized as follows. In Section 2 the algorithm and all its individual steps are described. The evaluation of its performance and comparison with existing spike detection methods are presented in Section 3. Conclusive remarks are given in Section 4. The notation used throughout this paper is explained in Table 1. 
TABLE 1: Bold lower case letters denote vectorial quantities whereas bold upper case letters represent matrices. Superscripts refer to the iteration index while subscripts refer to a group index.

\begin{tabular}{llc}
\hline Symbol & Description & Reference \\
\hline $\mathbf{q}_{i}$ & ith (true) waveform & Section 2.1 \\
$\mathbf{q}_{i}(t)$ & vector entry at $t$ th position & Section 2.1 \\
$\hat{\mathbf{q}}_{i}$ & estimate of the $i$ th waveform & Section 2.4 \\
$\mathbf{q}_{i}[t]$ & time dependent waveform & Section 3.8 \\
$\mathbf{C}, \widehat{\mathbf{C}}$ & true/estimated noise cov. matrix & Sections 2.1 and 2.6 \\
$\mathbf{h}^{(k)}$ & SEA filter at iteration $k$ & Section 2.2 \\
\hline
\end{tabular}

\section{Methods}

2.1. Model of Recorded Data. In order to derive a wellmotivated algorithm avoiding heuristics as much as possible, the recorded data have to be described by some signal model. In the neuroscience community, it is widely accepted that the data $\mathbf{x}$ recorded at an electrode can often be represented as a linear sum of convolutions of the intrinsic spike trains $\mathbf{s}_{i}$ with constant waveforms $\mathbf{q}_{i}$ and colored Gaussian noise n (having a noise covariance matrix $\mathbf{C}$ ); see, for example, $[26,27]$. Explicitly, it is

$$
\mathbf{x}(t)=\sum_{i=1}^{M} \sum_{\tau} \mathbf{q}_{i}(\tau) \mathbf{s}_{i}(t-\tau)+\mathbf{n}(t),
$$

where $M$ is the number of neurons whose spikes are present in the recordings. For the sake of clarity, we restricted the model to single channel recordings, that is, electrodes, but an extension to multichannel data as provided by tetrodes is straightforward.

Since the goal of spike detection is to recover the spike trains $\mathbf{s}_{i}$ from a linear time-invariant system without a priori knowledge about the shape of the waveforms $\mathbf{q}_{i}$, this can be viewed as a blind equalization problem (often also called blind deconvolution, blind identification, or convolutive blind source separation). An overview about this topic and a survey of available methods dealing with such problems can be found in [28].

Most often, $M$, the number of sources, will be larger than the number of recording channels. In the model of a single electrode as described in (1), the number of recording channels is equal to one, in which case the generative system is referred to as multiple-input, single-output. In general, it is not possible to extract more sources than available recording channels [28]. In the following, we make explicit use of the unique properties of neural data, such as sparseness and binary alphabet, to overcome this restriction partially.

2.2. Application of the Superexponential Algorithm. The superexponential algorithm (SEA) developed in [29] achieves blind equalization by applying a filter which is calculated by use of higher-order cross cumulants. For real-valued data, the filter $\mathbf{h}$ at iteration $k+1$ is computed as

$$
\mathbf{h}^{(k+1)}=\frac{\mathbf{R}^{-1} \cdot \mathbf{d}^{(k)}}{\sqrt{\mathbf{d}^{(k)^{\top}} \cdot \mathbf{R}^{-1} \cdot \mathbf{d}^{(k)}}},
$$

where $\mathbf{R}$ is the data covariance matrix, that is, $\mathbf{R}(i, j)=$ $\operatorname{cov}(\mathbf{x}(t-i), \mathbf{x}(t-j)), \mathbf{d}^{(k)}(n)=\operatorname{cum}\left(\mathbf{y}^{(k)}(t): p, \mathbf{x}(t-n):\right.$ 1) denotes the cross-cumulant between $p$-times $\mathbf{y}^{(k)}(t)$ and $\mathbf{x}(t-n)$ (hence, the order of the statistics is $p+1)$, and $\mathbf{y}^{(k)}(t)=\sum_{\tau} \mathbf{h}^{(k)}(\tau) \mathbf{x}(t+\tau)$ being the filter output.

The algorithm works when the signals $\mathbf{s}_{i}$ are nonGaussian and when the $\mathbf{q}_{i}$ are stable (stable in the sense of robust against noise, not in the sense of stationary in time). In the context of neural recordings, both requirements are surely met. Firstly, the $\boldsymbol{s}_{i}$ represent the intrinsic spike trains, thus taking values of either 0 or 1 , and whose probability density function follows most likely a sparse Bernoulli distribution, or their interspike interval a Poisson distribution. Secondly, the waveforms $\mathbf{q}_{i}$ are finite impulse response filters, and hence are stable. The SEA algorithm is said to have reached convergence when the difference between two consecutive iterations is small enough (see also Section 3.3). For convenience, we call the filter obtained at the last iteration simply $\mathbf{h}$, instead of $\mathbf{h}^{\left(k_{\text {last }}\right)}$.

The choice of the SEA instead of other blind equalization algorithms was motivated by several of its features. It is shown that in the noise-free case, the algorithm converges independently of the initial condition to the globally optimal solution with a superexponential convergence rate [29]. Since it uses higher-order statistics, this property should also hold approximatively when Gaussian noise is present, as higher-order cumulants are zero for Gaussian signals. Moreover, the algorithm is not gradient based like Bussgang type algorithms; thus no step size selection is required, which reduces the amount of parameter settings for the user.

For neural data, we chose the order of the cumulant to be $p=2$ or $p=3$. In the former case, the vector $\mathbf{d}$ is proportional to the skewness, a statistics which is well suited for asymmetric signals such as the $s_{i}$ [30]. For $p=3$, this makes the vector $\mathbf{d}$ proportional to the kurtosis, which is a good statistics in the case of sparse data following a model as in (1) [31]. These findings were also confirmed in [32].

2.3. Mode Detection in the SEA Filter Output. The SEA computes a single filter on the basis of a vector $\mathbf{d}$ which contains the statistics of all $M$ waveforms. Nevertheless, as it is most likely that the characteristics of the neurons will be different with respect to signal-to-noise ratio, spiking frequency, or shape of waveform, it is expected that the filter will have various responses to the different neuronal waveforms. The idea is to identify spikes which belong to a single component and recalculate the filter using only these spikes. The identification is done by a technique called mode finding [33]. Firstly, only the local maxima, denoted by $m_{i}$, of the filter output $y$ within a certain range $2 L_{s}+1$ are extracted. Then, the probability density function $p m$ of the $m_{i}$ is estimated by a kernel density estimator, which in the assumed case of Gaussian noise is favorable to be a Gaussian kernel. The kernel bandwidth is chosen optimally depending on the amount of data [34]. The function $p m$ will exhibit a high amplitude mode due to noise and possibly several low amplitude modes caused by spikes; see Figure 2. (Due to the large amount of noise samples, the kernel bandwidth 
will be relatively small, which guarantees that the modes caused by spikes will not be smoothed away.) Hence, the second largest mode, denoted by $b_{2}$, is the prominent spike mode, that is, caused by spikes to which the filter responded the most, and which consequently should be extracted from the data first (see also Section 2.3.3). All $m_{i}$ which have a smaller distance to $b_{2}$ than to any other spike mode, and which are also larger than the first local minimum separating the noise peak from the first spike mode, are considered to belong to $b_{2}$; see Figure 2 . However, modes which are in the range of $\pm 2 \hat{\sigma}_{n_{h}}$ around $b_{2}$ are not regarded as separate modes whereas $\hat{\sigma}_{n_{h}}$ denotes the estimated standard deviation of the noise in the filter output (of filter $\mathbf{h}$ ) (see Section 2.3.1). This is motivated by the fact that two Gaussian distributions with identical standard deviation do not exhibit two separate modes, unless their means are at least $2 \sigma_{n_{h}}$ apart [35]. This merging of modes is necessary in order to minimize the number of spurious modes which do not represent an individual component but are mere artifacts caused by the kernel smoothing.

2.3.1. Estimation of the Filter Output Noise Variance. To estimate $\sigma_{n_{h}}$, first the mean $\hat{\mu}_{n_{h}}$ of the filter output noise is estimated. If one can assume that the noise $\mathbf{n}$ is zero mean, this step can be avoided, since then it immediately follows that $\mu_{n_{h}}=0$ as well. Otherwise, the probability density function of $\mathbf{y}$ is estimated by a Gaussian kernel density estimator as described in the previous section. Making again use of the sparseness of the data, the mean $\hat{\mu}_{n_{h}}$ is found as the global maximum of this probability density function.

As we expect that the response of filter $\mathbf{h}$ to spikes is larger than $\mu_{n_{h}}$, we ignore all values of $\mathbf{y}$ which are above $\hat{\mu}_{n_{h}}$, since they are likely to contain spikes. Hence, $\hat{\sigma}_{n_{h}}$ is solely estimated on values of $\mathbf{y}$ which are smaller than $\hat{\mu}_{n_{h}}$.

2.3.2. Gaussianity of the Modes. Strictly speaking, due to the maximum operation, the $m_{i}$ do not follow a Gauss distribution anymore, but rather an extreme value distribution. Nevertheless, a Gaussian kernel is used for density estimation and the spike modes are assumed to be Gauss distributed as well. This is justified by the fact that the spike modes exhibit large amplitudes in the filter output, and thus their maximum values are still almost Gauss distributed even after a maximum operation.

2.3.3. Largest Spike Mode Finding. From the kernel density of the $m_{i}$, first a Gaussian distribution with mean $\hat{\mu}_{n_{h}}$ and standard deviation $\hat{\sigma}_{n_{h}}$ is subtracted (not shown in Figure 2). This removes the noise contribution to modes and ensures that the largest spike mode $b_{2}$ is indeed the prominent one.

Note that in [14] also a mode detection procedure was applied. In contrast to our approach, it was done on a generic filter output consisting of squaring and lowpass filtering. Moreover, we merge modes based on their proximity in order to find all spikes belonging to the largest spike mode, whereas in [14] only the local minimum separating the noise

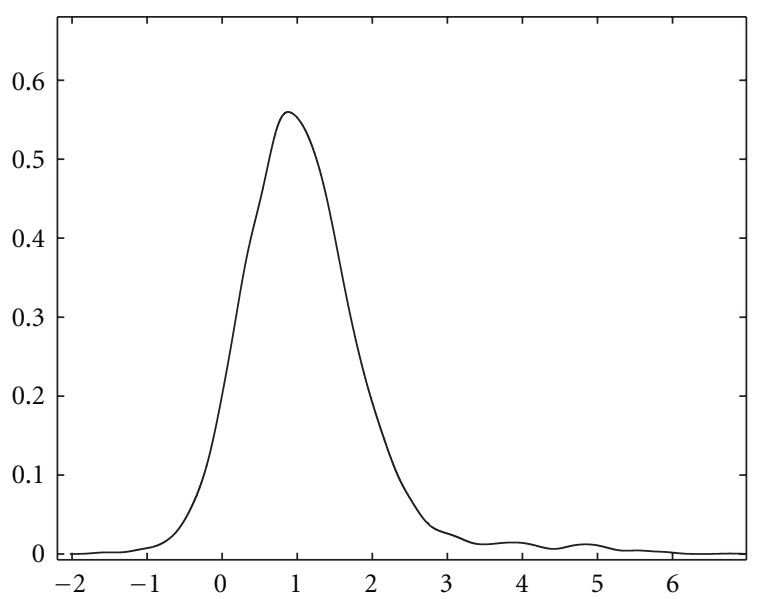

(a)

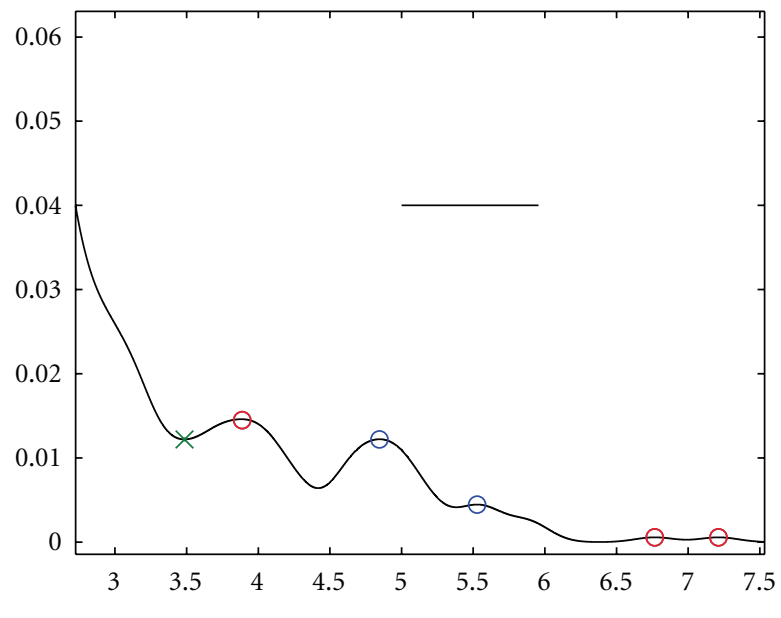

(b)

Figure 2: (a) Estimated probability density of the local maxima $m_{i}$. The spareness of the data is clearly exhibited by the large noise peak (at around 1 on the $x$-axis) and some small spike peaks (at around 4 and 5 on the $x$-axis). (b) Zoom in on the spike modes. The circles indicate the local maxima of the modes that were found. The mode at around 3.9 was identified as largest $\left(b_{2}\right)$, and the two modes indicated by blue circles are discarded, as they are within the range of $\pm 2 \hat{\sigma}_{n_{h}}$. The estimated noise standard deviation $\hat{\sigma}_{n_{h}}$ is indicated by the thick bar. The green cross indicates the first local minimum, separating the noise peak from the spike modes.

mode from the spike mode is found and a single template is constructed.

2.4. Sparse Deflation. In classical algorithms designed for multiple-input, multiple-output systems, sources are extracted one by one using a technique called deflation [36]. As such, one single waveform $\mathbf{q}_{j}$ is estimated via second order statistics, the source $\mathbf{s}_{j}$ is estimated via the convolution of the corresponding filter $\mathbf{h}_{j}$ with $\mathbf{x}$, and the convolution between $\mathbf{q}_{j}$ and $\mathbf{s}_{j}$ is subtracted from the data $\mathbf{x}$. This classical deflation procedure was developed by assuming that the sources are continuous signals and that the waveforms have to be known only up to a scalar factor. In contrast, the signals 
representing the occurrences of spikes are discrete and sparse, and, as will be shown in Section 2.6, the waveforms need to be known without ambiguity.

Therefore, we propose an adapted deflation procedure which we call sparse deflation, as it relies on the sparseness of the data. At iteration $j$ data segments $\mathbf{x}^{(j)}{ }_{i}$ of length $2 L_{f}+1$ are cut out of $\mathbf{x}$ around the occurrence times $t_{m_{i}}+t_{\text {shift }}$ of the local maxima $m_{i}, i=1, \ldots, K$, which belong to mode $b_{2}$. The shift $t_{\text {shift }}$ is determined so that the cut out data segments have maximum total energy. Without this step, extraction of different parts of the same waveform at several iterations would be possible, as the SEA filter does not necessarily respond maximally at the center of a waveform. Finally, the waveform is estimated as the median of all data segments, that is,

$$
\hat{\mathbf{q}}_{j}(t)=\operatorname{med}\left\{\mathbf{x}_{1}^{(j)}(t), \ldots, \mathbf{x}_{K}^{(j)}(t)\right\} \quad t=-L_{f}, \ldots, L_{f},
$$

where $K$ is the total number of local maxima $m_{i}$ belonging to mode $b_{2}$. (An even better performance could be achieved if the data segments were first upsampled, aligned, averaged, and then downsampled [27].) Instead of subtracting the estimated contribution of source $\boldsymbol{s}_{j}$, the data segments $\mathbf{x}^{(j)}{ }_{i}$ are simply removed from the data. The reduced dataset $\mathbf{x} \backslash \mathbf{x}^{(j)}{ }_{i}, i=1, \ldots, K$, is now used as the starting point for the next iteration of the algorithm. In particular, the steps described in Sections 2.2-2.4 are repeated on the updated data $\mathbf{x} \backslash \mathbf{x}^{(j)}{ }_{i=1, \ldots, K}=: \mathbf{x}$. (The covariance matrix $\mathbf{R}$ and cumulant vector $\mathbf{d}$ are computed on each remaining data chunk separately and the final estimates are obtained as a proportionally to the data chunk length weighted average.)

2.5. Abortion Criteria. The iteration loop (SEA, mode detection, sparse deflation) is terminated if at least one of the following criteria is met.

(i) No spike mode can be identified in the filter output anymore, or the number of spikes belonging to the spike mode $b_{2}$ is below a relative threshold $\min f$.

(ii) A maximum number of iterations is reached.

If the loop abortion happens after the first iteration already, the filter obtained by (2) is used for further spike detection instead of the MVDR beamformers.

2.6. Calculation of the MVDR Beamformers. Once the iteration loop described in the previous sections is completed, the final filters used for spike detection are calculated. Namely, we use the MVDR beamformers which are given by [18]

$$
\mathbf{f}_{i}=\frac{\hat{\mathbf{C}}^{-1} \cdot \hat{\mathbf{q}}_{i}}{\hat{\mathbf{q}}_{i}^{\top} \cdot \hat{\mathbf{C}}^{-1} \cdot \hat{\mathbf{q}}_{i}}
$$

where $\hat{\mathrm{C}}$ is the estimate of the noise covariance matrix, and $\hat{\mathbf{q}}_{i}$ denotes the vectorial representation of the $i$ th estimated waveform, the individual entries being $\hat{\mathbf{q}}_{i}\left(-T_{f}\right), \ldots, \hat{\mathbf{q}}_{i}\left(+T_{f}\right)$; (see (3)). (Note that other filters could be used instead, for example, adapted to a real-time detection task [37].) The MVDR beamformers are designed such that their response is one at the center of a waveform, that is, $\mathbf{f}_{i}^{\top} \cdot \hat{\mathbf{q}}_{i}=1$.

The estimate of $\mathbf{C}$ is done after the last algorithm iteration, as the deflated dataset $\mathbf{x} \backslash \mathbf{x}^{(j=1, \ldots, J)}{ }_{i=1, \ldots, K_{J}}$ contains far less spikes than the original data $\mathbf{x}$ allowing for a more accurate noise estimation.

2.7. Filtering and Spike Detection. After calculating the MVDR beamformers, the data are filtered with each of them, and a spike is declared as detected when the filter output $\mathbf{z}$ exceeds a certain threshold $\gamma$, that is,

$$
\begin{aligned}
\mathbf{z}_{j}(t) & =\sum_{\tau} \mathbf{f}_{j}(\tau) \mathbf{x}(t+\tau) \quad \text { detection } \quad \text { if } \mathbf{z}_{j}(t) \geq \gamma_{j} \\
& =:(\mathbf{f} \star \mathbf{x})(t) .
\end{aligned}
$$

2.8. Threshold Selection. The threshold for every filter is selected individually such that the probability of detection $P_{\mathrm{D}}$ is maximal (probability of a true positive detection), whereas the probability of false alarm $P_{\mathrm{FA}}$ (probability of a false positive detection) should be minimal. If one admits a certain tolerance $\Delta$ in the arrival time estimation, meaning that a spike is declared as correctly detected when the filter output exceeds the threshold somewhere in the interval $\left[t_{\text {spike }}-\Delta, t_{\text {spike }}+\Delta\right]$, the probability of detection for filter $\mathbf{f}_{j}$ given threshold $\gamma_{j}$ is expressed as

$$
P_{\mathrm{D} j}\left(\gamma_{j}\right)=1-\prod_{\tau=-\Delta}^{\Delta} P_{\mathrm{N} j}\left(\left(\mathbf{f}_{j} \star \overline{\mathbf{q}}_{j}\right)(\tau)\right),
$$

where $P_{\mathrm{N} j}(x):=1 / 2 \cdot\left(1+\operatorname{erf}\left(\left(\gamma_{j}-x\right) / \sqrt{2} \sigma_{j}\right)\right)$ with $\sigma_{j}:=$ $\sqrt{\mathbf{f}_{j}^{\top} \hat{\mathbf{C}} \mathbf{f}_{j}}$. Thus $P_{\mathrm{N}_{j}}\left(\left.x\right|_{x=\left(\mathbf{f}_{j} \star \overline{\mathbf{q}}_{j}\right)(\tau)}\right)$ is the probability that the waveform is not detected at time sample $\tau$, whereas $\overline{\mathbf{q}}_{j}$ is defined in Section 2.9. Similarly, the probability that a noise segment of length $2 \Delta+1$ is falsely detected is given by

$$
P_{\mathrm{FA} j}\left(\gamma_{j}\right)=1-\left(P_{\mathrm{N} j}(0)\right)^{2 \Delta+1} .
$$

An optimal detector would always achieve a perfect performance of $P_{\mathrm{D}}=1$ and $P_{\mathrm{FA}}=0$; thus any detector should have a performance as close as possible to the perfect performance. The optimal threshold, hence, is selected according to

$$
\gamma_{j}=\underset{\gamma_{j}}{\operatorname{argmin}}\left\{\left\|\left(\begin{array}{l}
0 \\
1
\end{array}\right)-\left(\begin{array}{c}
P_{\mathrm{FA} j}\left(\gamma_{j}\right) \\
P_{\mathrm{D} j}\left(\gamma_{j}\right)
\end{array}\right)\right\|\right\} .
$$

This optimization problem can be solved efficiently as it involves only a single parameter, namely, the threshold $\gamma_{j}$, which should lie in the interval $[0,1]$. In practice, we evaluate $P_{\mathrm{FA} j}$ and $P_{\mathrm{D} j}$ for all threshold values in $[0,1]$ with a resolution of 0.0005 , and select as optimal threshold the one which minimizes (8).

When the thresholds are obtained by (8), it is assumed that detecting a spike is equally important as avoiding a false positive detection. However, with respect to subsequent 
analysis for understanding the working principles of the nervous system, it was shown that not detecting a spike has more impact than declaring incorrectly a piece of noise as a spike [38]. This particular characteristic of neural data could be incorporated by introducing a weighting parameter in (8).

2.9. Adaptation to Changing Waveforms. In (1) we assumed that the waveforms $\mathbf{q}_{j}$ are constant in time, which is approximatively true for short periods at the beginning of an experiment. Due to tissue relaxation, however, the distance between the electrode and the neurons changes, which leads to altered recorded waveforms [24]. In [39] we proposed an adaptation scheme for an estimated spatial waveform and the corresponding filter. This method was especially designed for sparse binary data such as neuronal data. Herein, we shortly summarize this method and extend it to multiple, temporal waveforms. In brief, after every time interval $T$, each waveform is updated as the mean of the $K_{\text {opt }}$ last data chunks $\mathbf{r}$ of length $2 L_{f}+1$ which were detected as spikes, that is,

$$
\hat{\mathbf{q}}_{j}=\frac{1}{K_{\mathrm{opt}_{j}}} \cdot \sum_{i=K_{\max j}-K_{\mathrm{opt}_{j}}+1}^{K_{\max }} \mathbf{r}_{j, i}
$$

where $\mathbf{r}_{j, i}:=\left(\mathbf{x}\left(t(i)-L_{f}\right), \ldots, \mathbf{x}\left(t(i)+L_{f}\right)\right)^{\top}$ such that $\mathbf{f}_{j}^{\top} \cdot \mathbf{r}_{j, i} \geq \gamma_{j}$, and $K_{\max j}$ denotes the maximum number of found spikes by filter $\mathbf{f}_{j}$. If two or more filters detect the same spike, the spike is assigned to one filter only, namely, to the one which had a response closest to 1 . The optimal number of spikes for averaging is determined by

$$
K_{\mathrm{opt}_{j}}=\underset{K}{\operatorname{argmax}}\left\{M_{j}(K)\right\} \text {, }
$$

where $M:=P_{\mathrm{D} j}+\left(1-P_{\mathrm{FA} j}\right)$, and $\overline{\mathbf{q}_{j}}$ is estimated as the mean waveform of the $Q$ last detections of filter $\mathbf{f}_{j}$.

2.10. Implementation. The higher-order cross cumulants were calculated by the use of the HOSA toolbox [40]. The proposed algorithm was implemented in MATLAB version 7.6, but not optimized for maximum computational speed yet. The code and a sample file will be made available at the website http://user.cs.tu-berlin.de/ natora/.

Regarding computational complexity, the most expensive task is the computation of the cross cumulants during the SEA algorithm. This computation, however, can be parallelized, in the sense that every time shift can be computed on a separate computing unit.

\section{Performance Evaluation}

3.1. Generation of Artificial Data. Artificial data were generated according to the model in (1). The waveforms were constructed from sorted spikes obtained from acute recordings in the prefrontal cortex of macaque monkeys and had a length of about 0.9 millisecond; see Figures 3(a)$3(\mathrm{c})$. Detailed information about the sorting method and the experimental setup were described in $[41,42]$. The spike

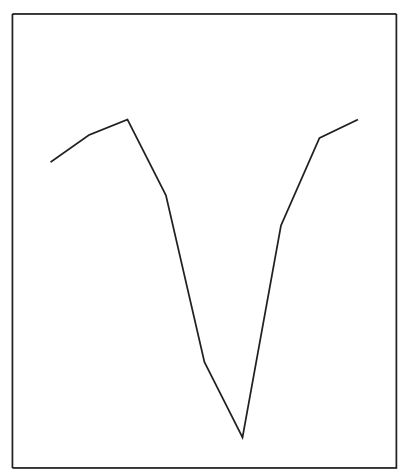

(a)

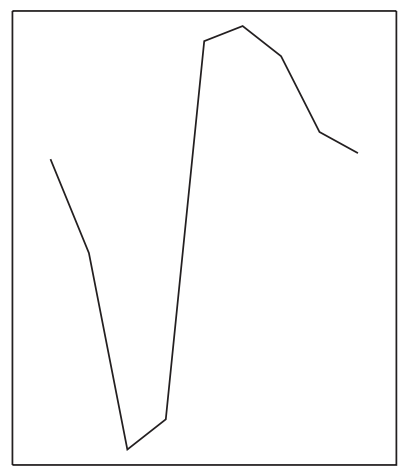

(c)

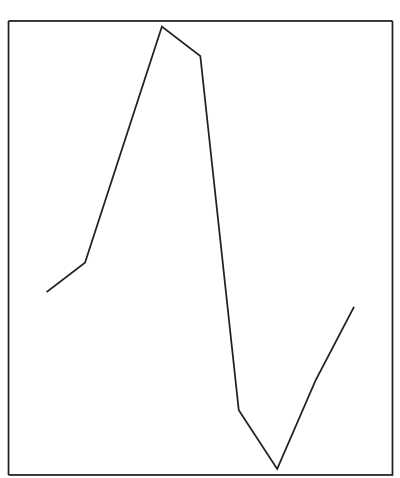

(b)

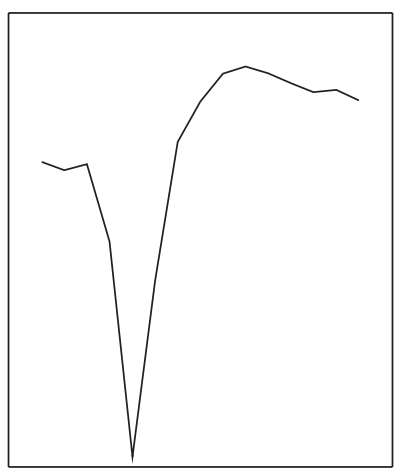

(d)
FIGURE 3: (a-c) Waveform templates obtained from extracellular recordings in macaque and used for generation of artificial datasets. (d) Waveform template obtained from simultaneous intra/extracellular recordings in rat tissue.

arrival times were simulated as independent homogeneous Poisson processes with an enforced refractory period of 2 millisecond. The noiseless data were simulated at a sampling frequency of $40 \mathrm{kHz}$ and then downsampled to $10 \mathrm{kHz}$, in order to include the phenomenon of sampling jitter as encountered in real recordings. Gaussian noise with an autocorrelation structure measured in real recordings was simulated by an ARMA process and added to the spike trains (see [42] for more details). Two types of datasets were simulated, one containing activity from two neurons, whereas the other one contained activity from three neurons. A data snapshot from the latter type is shown in Figure 4.

3.2. Performance Assessment. To allow for a better comparison, the most common definition of signal-to-noise ratio (SNR) utilized in the neuroscience community (see, e.g., [12]) was used. Namely, the SNR of the $i$-th spike train is defined as the ratio between the norm of the corresponding waveform and the standard deviation of noise:

$$
\mathrm{SNR}_{i}=\frac{\left\|\mathbf{q}_{i}\right\|_{\infty}}{\sigma_{n}} .
$$

The detection performance of an algorithm was investigated by means of receiver operator characteristic (ROC) 


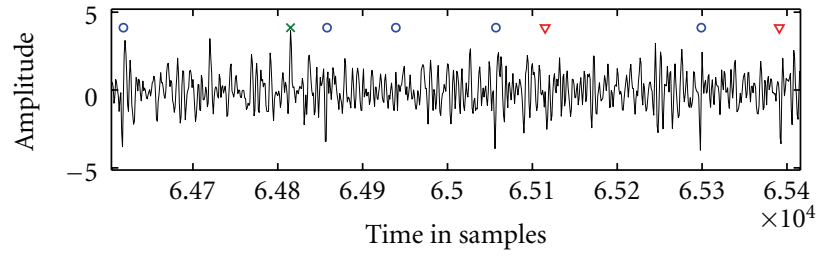

(a)

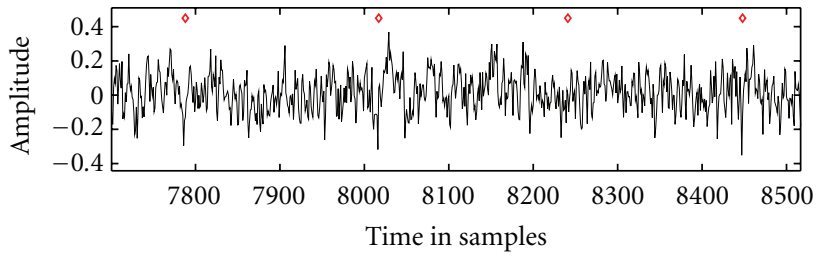

(b)

FIGURE 4: (a) Data chunk of simulated data with an SNR value of 3.0; that is, all inserted waveforms had an SNR of 3.0. The markers indicate the occurrence times of the inserted spikes, whereas the templates shown in Figures 3(a)-3(c), were used. (b) Data chunk of experimental data from simultaneous intra/extra-cellular recordings in rat tissue. The empirically determined SNR is 3.050 and the extracted waveform is shown in Figure 3(d).

curves and the corresponding areas under the curves (AUCs), similarly defined as in [14]. The ROC curves were calculated by evaluating the relative number of true positive (TP) and false positive detections (FP), given by

$$
\begin{aligned}
& \mathrm{TP}=\frac{\text { number of correct detections }}{\text { number of inserted spikes }} \\
& \mathrm{FP}=\frac{\text { number of false detections }}{\text { maximum number of possible false detections }} .
\end{aligned}
$$

A detection was classified as correct, if the detectors response was within \pm 0.4 millisecond of the true spiking time, which implied $\Delta=2$ in the parameter setting of the HBBSD algorithm. Multiple detections within this time frame were ignored. Consequently, there is a maximum number of possible false positive detections a detector can produce in a dataset of finite length. By the definition in (12), both quantities TP and FP are bounded on the interval $[0,1]$.

3.3. Parameter Settings of HBBSD. In all subsequent simulations the following parameters were used in the $H B B S D$ algorithm: the SEA algorithm was said to have reached convergence if $\left\|\mathbf{h}^{(k+1)}-\mathbf{h}^{(k)}\right\|_{2} \leq 10^{-10}$. The SEA algorithm used higher-order statistics with $p=2$ but switched automatically to $p=3$ if no convergence could be achieved in the former case. The minimum firing frequency $\min f$ was set to $5 \mathrm{~Hz}$, the filter length was equal to 9 samples $\left(L_{f}=L_{s}=4\right)$, and the maximum number of 3 filters was allowed. Here we would like to point out that, unlike in some other methods, where the parameters are algorithm specific and thus their value setting is not an obvious task, the parameters of $H B B S D$ are biologically motivated, allowing for a reasonable choice of their values. For example, since single channel data are analyzed, it is sound to assume that action potentials from not more than 3 to 4 nearby neurons will be recorded, justifying a maximum filter amount of 3 . The filter length can be chosen as the length of a spike, which is most often in the range of 0.4 to 1.0 millisecond [12]. Besides, there exist methods to estimate the filter length even when no biologically motivated a priori knowledge is available $[32,43]$. Finally, it is unlikely that neurons in a task relevant brain region will exhibit very low firing frequencies, but, as a matter of fact, the parameter $\min f$ could be dropped entirely from the algorithm structure.

The needed estimate of the waveform $\overline{\mathbf{q}}_{j}$ (see Section 2.9) was obtained as the mean of the $Q=75$ last detections. As was demonstrated in [39], the choice of the value for $Q$ is not critical.

3.4. Competing Algorithms. In [25] we compared the performance of $H B B S D$ with existing methods covering all categories described in Section 1. It was demonstrated that, in general, algorithms relying on waveform information outperform methods which are solely based on amplitude thresholding or transient detection. Finally, it was shown that a wavelet-based method such as [12] might perform poorly when the actual waveform is distinct from the used mother wavelet, and we concluded that the best available method at the time is the one presented in $[15,16]$. This method accomplishes blind equalization by cepstrum of bispectrum calculation and hence will be abbreviated as $C o B$. The parameters for this algorithm were set according to its reference and adapted to the herein considered sampling frequency and spike length. Additionally, we compared our method to the classical, single iteration, superexponential method, denoted by SEA.

3.5. Performance on Data with Two Waveforms. Ten independent simulations, each of 6 seconds in length, containing activity from two neurons with the waveforms (a) and (b) shown in Figure 3 were simulated. The spiking frequencies were $15 \mathrm{~Hz}$ and $25 \mathrm{~Hz}$, respectively.

In Figure 5 the results for all compared methods are shown. (This evaluation is quite short, since results on datasets containing one and two neurons were already presented in [25].) HBBSD achieves a clearly better performance than the competing methods, since it calculates several filters. When the threshold is selected automatically, the performance of $H B B S D$ often lies above the ROC curves (as, e.g., in Figure 5(b), or Figures 6(a) and 6(b)), since the threshold is selected for every filter individually, whereas for the ROC curves generation, the threshold is varied uniformly for all filters.

3.6. Performance on Data with Three Waveforms. Five independents simulations, each of 10 seconds in length, 


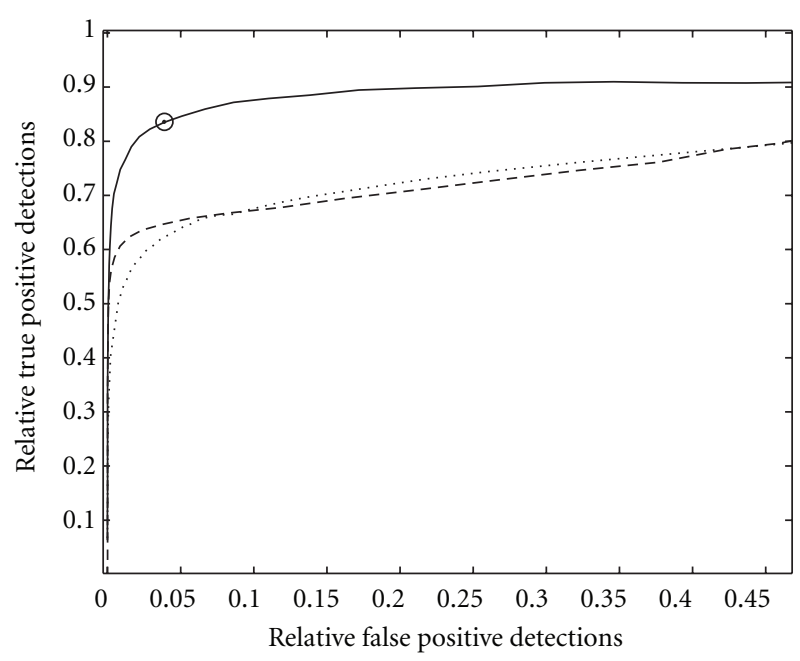

(a)

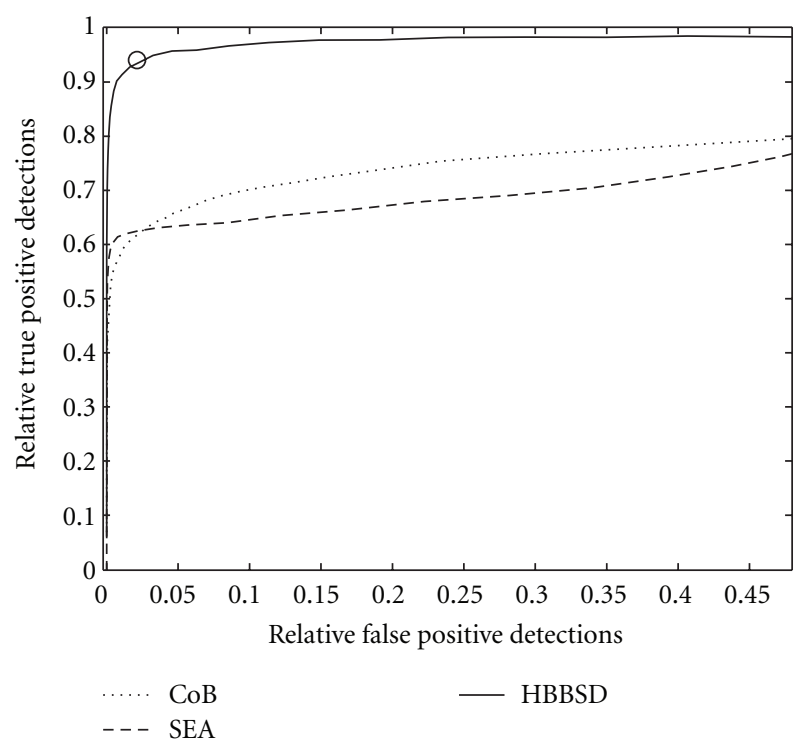

(b)

Figure 5: Average ROC curves for various spike detection methods on a dataset containing activity from two neurons. The shown results are an average over 10 independent simulations. (a) shows the results in the case of SNR $=3.25$, and (b) in the case of $\mathrm{SNR}=3.75$. The circle indicates the performance of the HBBSD algorithm when the threshold is selected automatically according to Section 2.8.

containing activity from three neurons with the three waveforms shown in Figures 3(a)-3(c), were simulated. The spiking frequencies were $15 \mathrm{~Hz}, 25 \mathrm{~Hz}$, and $20 \mathrm{~Hz}$, respectively. The SNR was varied from 3.0 to 4.25 in steps of 0.25 (all three spike trains always had equal SNR values), and again the ROC curves were computed for every method. To assess the overall performance for various SNR levels, the area under the ROC curves (AUC) was evaluated and is reported in Figure 6. Again, $H B B S D$ achieves the best performance throughout all SNR levels. The large standard deviation in the case of low SNR value
(Figure 6(c)) is explained by the fact that sometimes only one or two MVDR filters were calculated, since, due to the high noise, no further modes in the SEA output could be identified.

3.7. Performance on Simultaneous Intra/Extracellular Recordings. The same data as described in [42] were used; however, only single channel data were considered, and the data were downsampled to $10 \mathrm{kHz}$ for faster processing. For the evaluation we used two experiments in which each time a single cell from Long Evans rats (P17-P25) was stimulated by a current injection and simultaneously the extracellular potential was recorded. In one of the experiments, the total number of spikes was 244, and the SNR was empirically determined as 3.050 (a trace of this recording is shown in Figure 4, and the corresponding extracted waveform is shown in Figure 3(d)). Since the ground truth was known, the spikes were removed from the data, and higher-order statistics were calculated on the remaining noise samples indicating a skewness of -0.053 and an excess kurtosis of -0.161 . In the second experiment, a total of 103 spikes were found, the SNR being 3.008, the skewness being -0.012 , and the excess kurtosis being -0.295 . All the algorithms were applied to these real data with the same parameter settings as in the case of artificial data. The results are shown in Figure 7. As each experiment contained activity from only one cell, the performance gain of $H B B S D$ compared to the other methods is not that pronounced as on datasets containing several distinct waveforms. The results show, however, that $H B B S D$ is robust to violations of the assumptions made in the data model (1). Neither the skewness nor the excess kurtosis of the noise was equal to zero; nevertheless, the algorithm still achieved favorable results.

3.8. Performance on Nonstationary Data. Datasets with temporally changing waveforms were generated in the following manner. The first 8 seconds contained temporally constant waveforms and served as initialization data for the spike detection algorithms. Afterwards, the waveforms started to change for the next 2.5 minutes according to a normalized linear mixture (drift data), and finally in the last 50 seconds, again a constant waveform was present (end data). To sum up, the waveforms followed the model:

$$
\mathbf{q}[t]= \begin{cases}\mathbf{q}_{i_{1}}, & \forall t \leq 8 \mathrm{~s}, \\ \alpha_{i_{3}}[t] \cdot \mathbf{q}_{i_{3}}[t], & \forall t \in[8 \mathrm{~s}, 158 \mathrm{~s}], \\ \mathbf{q}_{i_{2}}, & \forall t \geq 150 \mathrm{~s},\end{cases}
$$

where $\mathbf{q}_{i_{3}}[t]:=\left(\mathbf{q}_{i_{2}}-\mathbf{q}_{i_{1}}\right) / 150 \mathrm{~s} \cdot t+158 \cdot \mathbf{q}_{i_{1}}-8 \cdot \mathbf{q}_{i_{2}} / 150$. (In order to distinguish the time dependent waveforms from the notation in previous sections where the time index referred to a vector entry, the notation $\mathbf{q}[t]$ is used here.) The value of $\alpha_{i_{3}}[t]$ is set so that the SNR value stays constant all the time. Two different scenarios were simulated. In the first one, the data contained a $25 \mathrm{~Hz}$ firing neuron, whose waveform had an SNR of 3.5 and changed from waveform (b) to waveform (a) as shown in Figure 3. In the second scenario, 


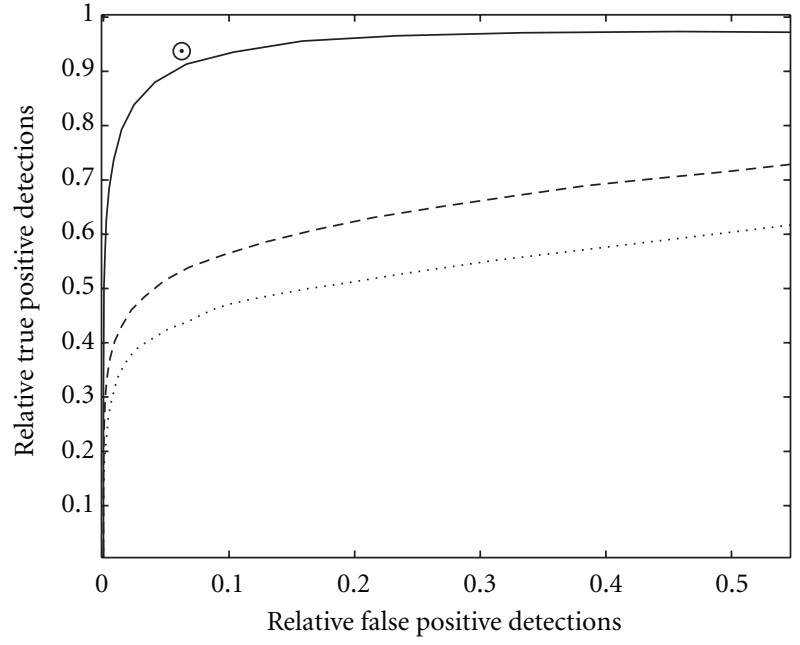

(a)

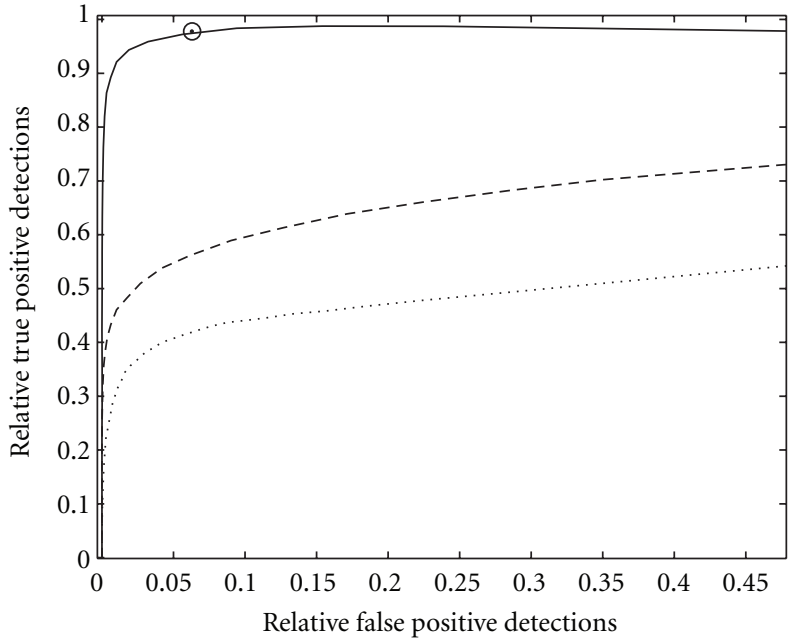

(b)

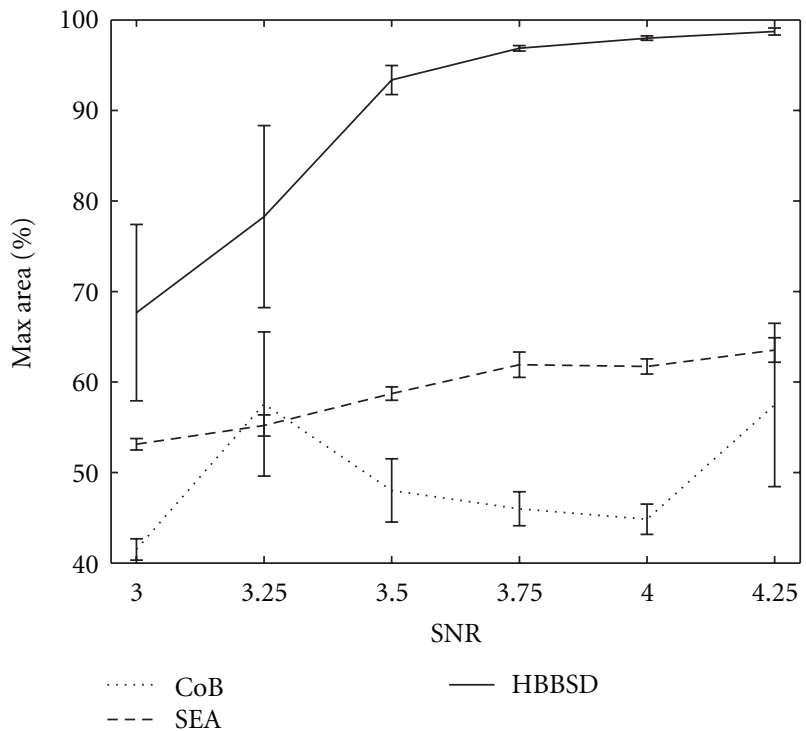

(c)

Figure 6: Average ROC curves for various spike detection methods on a dataset containing activity from three neurons. The shown results are an average over 5 independent simulations. (a) and (b) show the performance for SNR values of 3.5 and 4.0, respectively. The circle indicates the performance of $H B B S D$ when the threshold is selected automatically. (c) shows the relative area under the ROC curves and the corresponding standard deviations for several SNR levels.

data containing two neurons firing at $15 \mathrm{~Hz}$ and $25 \mathrm{~Hz}$, respectively, were simulated. The waveform of one neuron changed from the waveform (b) to waveform (a), whereas the waveform of the second neuron changed from waveform A to waveform (c) as shown in Figure 3.

The filters of the HBBSD method were adapted as described in Section 2.9, and the thresholds as described in Section 2.8. The adaptation was performed after every $T=5$ seconds. For comparison to nonadaptive methods, the MVDR filter from the SEA algorithm applied on the initialization data was calculated and used for spike detection on the drift and end data. The threshold was also kept constant to the value obtained on the initialization data by the method described in Section 2.8 (this method is still denoted by SEA in Figure 8, since it relies on a single filter). Similarly the filter computed by the $C o B$ method on the initialization data was used for spike detection on all subsequent data segments. The threshold was set to the default value of $0.04 \cdot k_{i}$, where $k_{i}$ denotes the maximum value of the filter output on the $i$-th data segment [15]. The performance of the algorithm was evaluated with respect to the relative total error TE which is defined as

$$
\mathrm{TE}=\frac{\mathrm{FP}+(1-\mathrm{TP})}{2}
$$




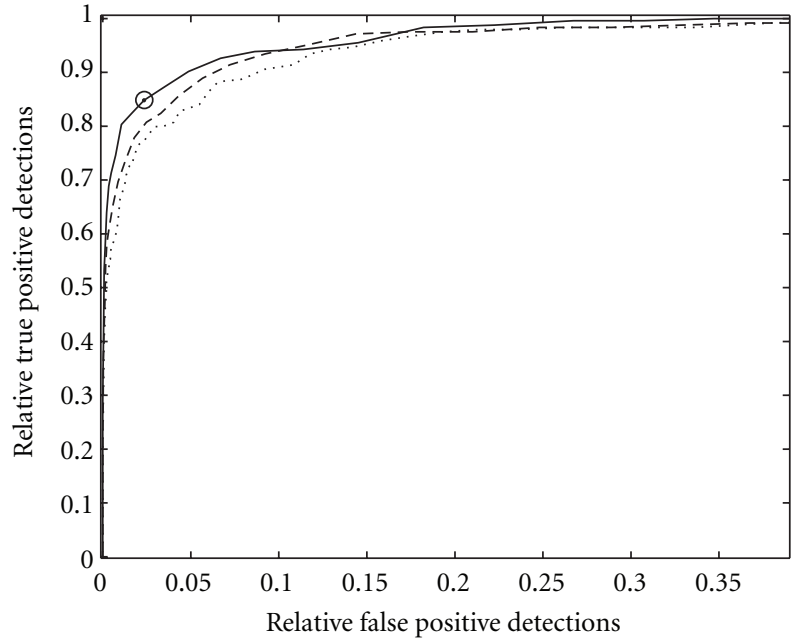

(a)

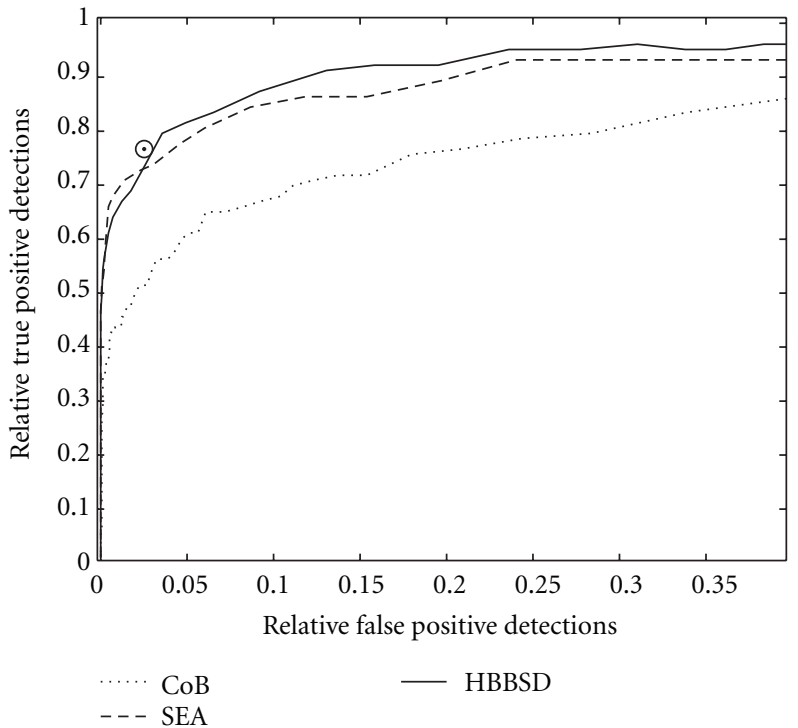

(b)

FIGURE 7: ROC curves for various spike detection methods on two experiments from simultaneous intra/extra-cellular recordings of cells in rat slices. The circle indicates the performance of HBBSD when the threshold is selected automatically. (a) Performance on a dataset with an empirical SNR value of 3.050 containing 244 spikes. (b) Performance on a dataset with an empirical SNR value of 3.008 containing 103 spikes.

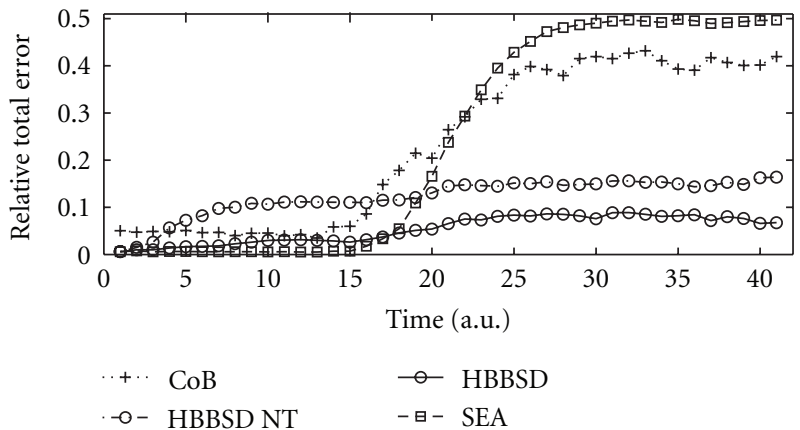

(a)

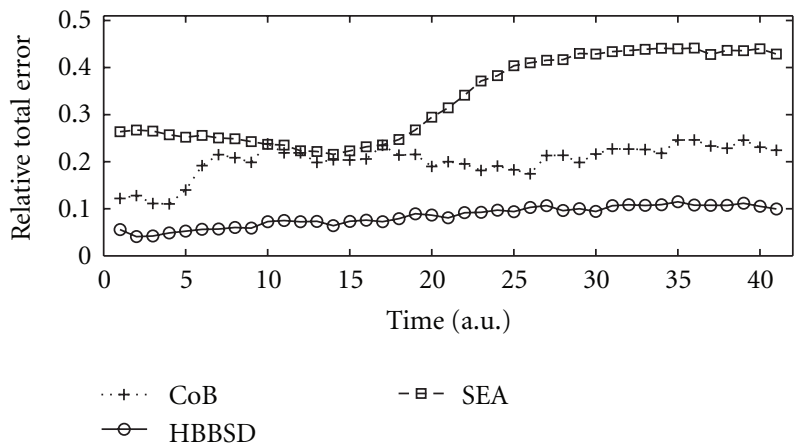

(b)

FIGURE 8: Average relative total error of various spike detection methods in the case of nonstationary waveform templates. The shown results are an average over 10 independent simulations. (a) Data containing a single, temporally changing waveform. (b) Data containing two, temporally changing waveforms.

where FP and TP are given by (12). The worst possible detector would have a score of $\mathrm{TE}=1$; the score for any reasonable detector, however, should not exceed $\mathrm{TE}=0.5$, as it either detects all spikes and generates a lot of false positive detections or vice versa.

The results for both scenarios are shown in Figure 8. The HBBSD algorithm was run in one of the scenarios without adapting the threshold, which is denoted by $H B B S D$ NT. Clearly, the adaptive algorithms achieve much better performance than the static methods, whereas the fully adaptive $H B B S D$ scores best. $C O B$ achieves in general a better performance than $S E A$, because the threshold is data driven (i.e., a relative value of the maximum filter output amplitude), while on the other hand a fixed absolute value for $S E A$ was used.

\section{Conclusion}

To our knowledge, blind equalization algorithms relying on higher-order statistics have rarely been applied to the task of neural spike detection. In this work, the superexponential algorithm has been used for initial filter estimation. Furthermore, a mode detection and a sparse deflation procedure have been proposed in order to extract multiple spike waveforms allowing to construct MVDR beamformers 
which offer a better detection performance than the SEA filters.

To sum up, a novel method for unsupervised spike detection has been presented, which relies on the inherent characteristics of data from neural recordings, such as sparseness and binary sources. For instance, the sparseness of the neuronal signal was exploited for mode finding in the filter output and for proposing a sparse deflation procedure which reduces error propagation. On the other hand, the binary source property allowed for an appropriate choice of the statistics for the SEA algorithm as well as for an easy estimation of the waveforms and construction of the MVDR filters.

In contrast to existing blind devonvolution methods which assume a finite alphabet or binary sources such as [44-47], we also made use of the spareness property and formulated a statistical algorithm (as opposed to deterministic/algebraic ones) which does not rely on extensive optimization of some cost functions. On the other hand, existing approaches dealing with sparse signals often assume instantaneous mixtures or apply a corresponding transformation into the frequency domain [48] or use clustering techniques together with further assumptions about the data (like high SNR) [49]. In this contribution, we operated always in the time domain whereby no further assumptions had to be made about the data. Moreover, we focused on the task of spike detection; thus, the complete separation of all sources is not required as it is in the existing approaches. The special structure induced by spareness and convolutive filters is currently still being investigated and only first attempts have been made to fully incorporate it into algorithm design $[50,51]$.

The main advantage of our method, namely, that several data-driven filters are calculated, resulted in a superior performance of $H B B S D$ compared to wavelet methods or other existing blind equalization algorithms. Furthermore, since the waveforms are estimated, this could be used as an initialization for a spike sorting algorithm, for example, using the idea of [52]. On the basis of waveform estimation, we also proposed a procedure for optimal threshold selection and drift adaptation. Especially the latter one again relies on the distinct properties of neural data.

The whole algorithm was tested on various datasets and compared to current state-of-the-art spike detection techniques. The used data covered not only simulated datasets containing two or three distinct waveforms but also experimental data containing a single waveform. In all these different conditions the proposed algorithm worked well and delivered better performance than the competing methods.

In order to allow for further evaluation and application the proposed algorithm will be made available online to the community at the website http://user.cs.tu -berlin.de/ natora/.

\section{Acknowledgments}

This research was supported by the Federal Ministry of Education and Research (BMBF) with the Grant 01GQ0743 and by the German Research Foundation (DFG) with the Grant Grk1589. The authors would like to thank Professor Aapo Hyvärinen for helpful discussion, and Małgorzata M. Wójcik for help with proof-reading.

\section{References}

[1] I. Obeid and P. D. Wolf, "Evaluation of spike-detection algorithms for a brain-machine interface application," IEEE Transactions on Biomedical Engineering, vol. 51, no. 6, pp. 905$911,2004$.

[2] A. Maccione, M. Gandolfo, P. Massobrio, A. Novellino, S. Martinoia, and M. Chiappalone, "A novel algorithm for precise identification of spikes in extracellularly recorded neuronal signals," Journal of Neuroscience Methods, vol. 177, no. 1, pp. 241-249, 2009.

[3] T. Borghi, R. Gusmeroli, A. S. Spinelli, and G. Baranauskas, "A simple method for efficient spike detection in multiunit recordings," Journal of Neuroscience Methods, vol. 163, no. 1, pp. 176-180, 2007.

[4] S. Mukhopadhyay and G. C. Ray, "A new interpretation of nonlinear energy operator and its efficacy in spike detection," IEEE Transactions on Biomedical Engineering, vol. 45, no. 2, pp. 180-187, 1998.

[5] J. H. Choi and T. Kim, "Neural action potential detector using multi-resolution TEO," Electronics Letters, vol. 38, no. 12, pp. 541-543, 2002.

[6] J. H. Choi, H. K. Jung, and T. Kim, "A new action potential detector using the MTEO and its effects on spike sorting systems at low signal-to-noise ratios," IEEE Transactions on Biomedical Engineering, vol. 53, no. 4, pp. 738-746, 2006.

[7] C. Paterson, R. Curry, A. Purvis, and S. Johnson, "Detection of action potentials in the presence of noise using phasespace techniques," World Academy of Science, Enineering and Technology, vol. 44, pp. 76-79, 2008.

[8] N. Mtetwa and L. S. Smith, "Smoothing and thresholding in neuronal spike detection," Neurocomputing, vol. 69, no. 10-12, pp. 1366-1370, 2006.

[9] T. I. Aksenova, O. K. Chibirova, O. A. Dryga, I. V. Tetko, A. L. Benabid, and A. E. P. Villa, "An unsupervised automatic method for sorting neuronal spike waveforms in awake and freely moving animals," Methods, vol. 30, no. 2, pp. 178-187, 2003.

[10] Z. Tiganj and M. Mboup, "Spike detection and sorting: combining algebraic differentiations with ICA," in Proceedings of the 8th International Conference on Independent Component Analysis and Signal Separation (ICA '09), vol. 5441 of Lecture Notes in Computer Science, pp. 475-482, March 2009.

[11] K. H. Kim and S. J. Kim, "A wavelet-based method for action potential detection from extracellular neural signal recording with low signal-to-noise ratio," IEEE Transactions on Biomedical Engineering, vol. 50, no. 8, pp. 999-1011, 2003.

[12] Z. Nenadic and J. W. Burdick, "Spike detection using the continuous wavelet transform," IEEE Transactions on Biomedical Engineering, vol. 52, no. 1, pp. 74-87, 2005.

[13] S. Dandapat and G. C. Ray, "Spike detection in biomedical signals using midprediction filter," Medical and Biological Engineering and Computing, vol. 35, no. 4, pp. 354-360, 1997.

[14] S. Kim and J. McNames, "Automatic spike detection based on adaptive template matching for extracellular neural recordings," Journal of Neuroscience Methods, vol. 165, no. 2, pp. 165174, 2007. 
[15] S. Shahid and L. S. Smith, "A novel technique for spike detection in extracellular neurophysiological recordings using cepstrum of bispectrum," in Proceedings of the 16th European Signal Processing Conference(EUSIPCO '08), 2008.

[16] S. Shahid, J. Walker, and L. S. Smith, "A new spike detection algorithm for extracellular neural recordings," IEEE Transactions on Biomedical Engineering, vol. 57, no. 4, Article ID 5166520, pp. 853-866, 2010.

[17] A. Zviagintsev, Y. Perelman, and R. Ginosar, "Algorithms and architectures for low power spike detection and alignment," Journal of Neural Engineering, vol. 3, no. 1, pp. 35-42, 2006.

[18] H. L. V. Trees, Detection, Estimation, and Modulation Theory Part IV-Optimum Array Processing, John Wiley \& Sons, New York, NY, USA, 2002.

[19] C. M. Gray, P. E. Maldonado, M. Wilson, and B. McNaughton, "Tetrodes markedly improve the reliability and yield of multiple single-unit isolation from multi-unit recordings in cat striate cortex," Journal of Neuroscience Methods, vol. 63, no. 1-2, pp. 43-54, 1995.

[20] P. T. Watkins, G. Santhanam, K. V. Shenoy, and R. R. Harrison, "Validation of adaptive threshold spike detector for neural recording," in Proceedings of the Annual International Conference of the IEEE Engineering in Medicine and Biology (IEMBS '04), vol. 2, pp. 4079-4082, September 2004.

[21] L. Traver, C. Tarín, P. Martí, and N. Cardona, "Adaptivethreshold neural spike detection by noise-envelope tracking," Electronics Letters, vol. 43, no. 24, pp. 1333-1335, 2007.

[22] H. L. Chan, M. A. Lin, T. Wu, S. T. Lee, Y. T. Tsai, and P. K. Chao, "Detection of neuronal spikes using an adaptive threshold based on the max-min spread sorting method," Journal of Neuroscience Methods, vol. 172, no. 1, pp. 112-121, 2008.

[23] E. Biffi, D. Ghezzi, A. Pedrocchi, and G. Ferrigno, "Development and validation of a spike detection and classification algorithm aimed at implementation on hardware devices," Computational Intelligence and Neuroscience, vol. 2010, Article ID 659050, 2010.

[24] E. A. Branchaud, J. W. Burdick, and R. A. Andersen, "An algorithm for autonomous isolation of neurons in extracellular recordings," in Proceedings of the 1st IEEE/RASEMBS International Conference on Biomedical Robotics and Biomechatronics (BioRob '06), pp. 939-945, February 2006.

[25] M. Natora, F. Franke, and K. Obermayer, "Spike detection in extracellular recordings by hybrid blind beamforming," in Proceedings of the 32nd Annual International Conference of the IEEE Engineering in Medicine and Biology Society (EMBS '10), pp. 4636-4641, 2010.

[26] M. Sahani, J. S. Pezaris, and R. A. Andersen, "On the separation of signals from neighboring cells in tetrode recordings," in Proceedings of the 10th Conference on Advances in Neural Information Processing Systems (NIPS '98), pp. 222-228, MIT Press, Cambridge, Mass, USA, 1998.

[27] C. Pouzat, O. Mazor, and G. Laurent, "Using noise signature to optimize spike-sorting and to assess neuronal classification quality," Journal of Neuroscience Methods, vol. 122, no. 1, pp. 43-57, 2002.

[28] C. Y. Chi, C. Y. Chen, C. H. Chen, and C. C. Feng, "Batch processing algorithms for blind equalization using higherorder statistics," IEEE Signal Processing Magazine, vol. 20, no. 1, pp. 25-49, 2003.

[29] O. Shalvi and E. Weinstein, "Super-exponential methods for blind deconvolution," IEEE Transactions on Information Theory, vol. 39, no. 2, pp. 504-519, 1993.
[30] P. Pjrvi, Adaptive blind deconvolution using third-order moments: exploiting asymmetry, Ph.D. thesis, Lulea University of Technology, Lulea, Sweden, 2005.

[31] J. Karvanen and A. Cichocki, "Measuring sparseness of noisy signals," in Proceedings of the 4th International Symposium on Independent Component Analysis and Blind Signal Separation (ICA '03), pp. 125-130, 2003.

[32] Y. Li, P. W. Tse, and X. Wang, "Recovery of vibration signal based on a super-exponential algorithm," Journal of Sound and Vibration, vol. 311, no. 1-2, pp. 537-553, 2008.

[33] M. Á. Carreira-Perpiñán, "Mode-finding for mixtures of gaussian distributions," IEEE Transactions on Pattern Analysis and Machine Intelligence, vol. 22, no. 11, pp. 1318-1323, 2000.

[34] M. C. Jones, J. S. Marron, and S. J. Sheather, "A brief survey of bandwidth selection for density estimation," Journal of the American Statistical Association, vol. 91, no. 433, pp. 401-407, 1996.

[35] K. Roeder, "A graphical technique for determining the number of components in a mixture of normals," Journal of the American Statistical Association, vol. 89, no. 426, pp. 487-495, 1994.

[36] Y. Inouye and K. Tanebe, "Super-exponential algorithms for multichannel blind deconvolution," IEEE Transactions on Signal Processing, vol. 48, no. 3, pp. 881-888, 2000.

[37] M. Natora, F. Franke, and K. Obermayer, "Optimal convolutive filters for real-time detection and arrival time estimation of transient signals," Proceedings of World Academy of Science, Engineering and Technology, vol. 55, pp. 235-240, 2009.

[38] A. Pazienti and S. Grün, "Robustness of the significance of spike synchrony with respect to sorting errors," Journal of Computational Neuroscience, vol. 21, no. 3, pp. 329-342, 2006.

[39] M. Natora, F. Franke, S. A. Broda, and K. Obermayer, "Optimal steering vector adaptation for linear filters leading to robust beamforming," in Proceedings of the 4th International Symposium on Communications, Control, and Signal Processing, (ISCCSP '10), pp. 1-6, March 2010.

[40] A. Swami, J. M. Mendel, and C. L. Nikias, Higher-Order Spectral Analysis Toolbox User's Guide, United Signal and Systems, Inc., 2001.

[41] M. Natora, F. Franke, M. Munk, and K. Obermayer, "Blind source separation of sparse overcomplete mixtures and application to neural recordings," in Proceedings of the 8th International Conference on Independent Component Analysis and Signal Separation, vol. 5441 of Lecture Notes in Computer Science, pp. 459-466, March 2009.

[42] F. Franke, M. Natora, C. Boucsein, M. H. J. Munk, and K. Obermayer, "An online spike detection and spike classification algorithm capable of instantaneous resolution of overlapping spikes," Journal of Computational Neuroscience, vol. 29, no. 12, pp. 127-148, 2010.

[43] F. Riera-Palou, J. M. Noras, and D. G. M. Cruickshank, "Segmented equalizers with dynamic length selection," in Proceedings of the 35th Asilomar Conference on Signals, Systems and Computers (ASILOMAR '01), vol. 2, pp. 951-955, November 2001.

[44] D. Yellin and B. Porat, "Blind identification of FIR systems excited by discrete-alphabet inputs," IEEE Transactions on Signal Processing, vol. 41, no. 3, pp. 1331-1339, 1993.

[45] T. H. Li, "Finite-alphabet information and multivariate blind deconvolution and identification of linear systems," IEEE Transactions on Information Theory, vol. 49, no. 1, pp. 330337, 2003.

[46] Y. Li, A. Cichocki, and L. Zhang, "Blind source estimation of FIR channels for binary sources: a grouping decision 
approach," Signal Processing, vol. 84, no. 12, pp. 2245-2263, 2004.

[47] K. I. Diamantaras and T. Papadimitriou, "Blind deconvolution of multi-input single-output systems with binary sources," IEEE Transactions on Signal Processing, vol. 54, no. 10, pp. 3720-3731, 2006.

[48] P. D. O'Grady, B. A. Pearlmutter, and S. T. Rickard, "Survey of sparse and non-sparse methods in source separation," International Journal of Imaging Systems and Technology, vol. 15, no. 1, pp. 18-33, 2005.

[49] D. Luengo, I. Santamaría, and L. Vielva, "A general solution to blind inverse problems for sparse input signals," Neurocomputing, vol. 69, no. 1-3, pp. 198-215, 2005.

[50] F. Marvasti, A. Amini, F. Haddadi et al., "A unified approach to sparse signal processing," CoRR. In press.

[51] X. F. Xu, D. Z. Feng, W. X. Zheng, and H. Zhang, "Convolutive blind source separation based on joint block Toeplitzation and block-inner diagonalization," Signal Processing, vol. 90, no. 1, pp. 119-133, 2010.

[52] Z. Koldovský and P. Tichavský, "Time-domain blind audio source separation using advanced component clustering and reconstruction," in Proceedings of the Hands-free Speech Communication and Microphone Arrays (HSCMA '08), pp. 216219,2008 